Research Paper

\title{
High Intratumoral Expression of Tetranectin Associates with Poor Prognosis of Patients with Gastric Cancer after Gastrectomy
}

\author{
Hao Chen ${ }^{1 *}$, Haojie $\mathrm{Li}^{1 *}$, Junjie Zhao ${ }^{1 *}$, Peike Peng ${ }^{2,3}$, Miaomiao Shao ${ }^{2,3}$, Hao Wu ${ }^{2,3}$, Xuefei Wang1, Ling \\ Chen $^{1}$, Qi Zhang ${ }^{1}$, Yuanyuan Ruan ${ }^{2,3 凶}$, Fenglin Liu $^{1 凶}$, Yihong Sun ${ }^{1 凶}$ \\ 1. Department of General Surgery, Zhongshan Hospital, Fudan University, Shanghai 200032, China; \\ 2. Department of Biochemistry and Molecular Biology, School of Basic Medical Sciences, Fudan University, Shanghai 200032, P.R. China; \\ 3. Key Laboratory of Glycoconjugate Research Ministry of Public Health, School of Basic Medical Sciences, Fudan University, Shanghai 200032, P.R. China. \\ * These authors contributed equally to this work. \\ $\square$ Corresponding authors: Fenglin Liu, M.D., Department of General Surgery, Zhongshan Hospital, Fudan University, Shanghai 200032, P. R. China. Tel: \\ 86-21-64041990-2910. Fax: 86-21-64041990-2910. Email: Liu.fenglin@zs-hospital.sh.cn. Or Yihong Sun, M.D., Department of General Surgery, Zhongshan \\ Hospital, Fudan University, Shanghai 200032, P. R. China. Tel: 86-21-64041990-2910. Fax: 86-21-64041990-2910. Email: sun.yihong@zs-hospital.sh.cn. Or \\ Yuanyuan Ruan, Ph.D., Department of Biochemistry and Molecular Biology, School of Basic Medical Sciences, Fudan University, 138 Yixueyuan Road, Shanghai \\ 200032, P. R. China. Tel: +86-21-54237795. Fax: +86-21-64437703. E-mail: yuanyuanruan@fudan.edu.cn.
}

(c) Ivyspring International Publisher. This is an open access article distributed under the terms of the Creative Commons Attribution (CC BY-NC) license (https://creativecommons.org/licenses/by-nc/4.0/). See http://ivyspring.com/terms for full terms and conditions.

Received: 2017.02.01; Accepted: 2017.08.31; Published: 2017.10.09

\begin{abstract}
Tetranectin, encoded by the clec $3 b$ gene, is a plasminogen kringle- 4 binding protein that can be detected in the plasma and the extracellular matrix. In malignancies, tetranectin is thought to enhance proteolytic processes enabling tumor cells to invade and metastasize. Nevertheless, the prognostic value of tetranectin in gastric cancer remains elusive. In this study, we found the expression of tetranectin was decreased in gastric cancer. High intratumoral tetranectin level was positively associated with tumor invasion $(P=0.013)$, lymph node metastasis $(P=0.005)$, advanced TNM stage $(P=0.003)$ and shorter overall survival $(O S)(P<0.001)$ for patients with gastric cancer. Tetranectin expression was identified as an independent prognostic factor for poor OS, and combining tetranectin expression with other independent prognostic factors generated a predictive nomogram, which showed better prognostic efficiency for OS in patients with gastric cancer. In summary, our study suggests that intratumoral tetranectin is a potential independent unfavorable prognostic biomarker for OS of patients with gastric cancer after gastrectomy.
\end{abstract}

Key words: Gastric cancer; Tetranectin; Prognosis; Nomogram.

\section{Introduction}

Gastric cancer is an important health problem, being the fifth most common cancer and the third leading cause of cancer-related death worldwide [1]. More than 950,000 new diagnoses are made every year [2]. In China, although the incidence has decreased over past several decades, gastric cancer still ranks the second most common malignancy with an estimated 679,100 new cases each year[3]. Due to the lack of specific symptoms at the early stage, over $80 \%$ of patients with gastric cancer are diagnosed at an advanced and unsuitable stage for surgical resection, which is the major reason for a poor prognosis [3]. Tumor-node-metastasis (TNM) staging system has been widely used to provide reliable prognostic information of gastric cancer, but they still have limited capacity to determine different outcomes for ignoring the heterogeneity of gastric cancer. Considering the increasing level of understanding of the molecular basis of tumor biology, more novel biomarkers are applied in diagnosis, prognosis and treatment [4].

Tetranectin, a homotrimeric C-type lectin 
originally isolated from plasma, was detected to bind with plasminogen and fibrin [5]. It is thought to play an important role in the regulation of proteolytic processes via binding to plasminogen [5]. Impaired cutaneous wound healing, delayed fracture healing and features of Parkinson disease were found in mice lacking tetranectin [6-8]. In malignancies, preoperative serum tetranectin was supposed to be a significant prognostic marker in advanced ovarian cancer patients [9]. The alteration of tetranectin expression in cancer tissue is also considered as a potential biomarker predicting prognosis in breast [10], bladder [11], oral [12] and ovarian cancer [13]. Nevertheless, the roles of tetranectin in gastric carcinoma remain poorly understood.

The aim of the present study was to investigate the expression of tetranectin in gastric cancer specimens and explore its associations with clinicopathological factors and prognosis. We also evaluated whether integration of intratumoral tetranectin expression could generate a predictive nomogram to refine the risk stratification system for prognosis of patients with gastric cancer.

\section{Materials and Methods}

\section{Patient samples}

A total of 328 consecutive gastric cancer patients who received standard gastrectomy with D2 dissection from the same surgical team in Zhongshan Hospital of Fudan University (Shanghai, China) between May 2002 and April 2006 were enrolled in the study. We retrospectively collected the baseline demographic and clinicopathological factors of these patients, including age, gender, tumor location, tumor differentiation, Lauren classification, and tumor stage. Tumor stage and tumor differentiation were reassessed by two independent gastroenterology pathologists according to the 2010 International Union Against Cancer TNM classification system. All patients were followed up until July 2012, with a median follow-up time of 59 months. OS was defined as the time between the dates of surgery and death or last visit. No patients received any preoperative anticancer treatment. All methods were approved by the research medical ethics committee of Fudan University and were carried out in accordance with the approved guidelines. Informed consent on the use of clinical specimens were obtained from all patients.

\section{TCGA and GEO datasets}

These data are publically available from the Cancer Genome Atlas and the NCBI Gene Expression Omnibus (accession number: GSE27342 and GSE13911). For the TCGA dataset, all level-3 data were downloaded from the TCGA-STAD portal by using TCGA-Assembler software[14]. The mRNA expression in TCGA dataset was measured by RNA sequencing V2. The RSEM (RNA-Seq by Expectation-Maximization) counts were further normalized by TMM (trimmed mean of $M$ value) method to estimate the relative RNA production levels using edgeR software[15]. For the Gene Expression Omnibus dataset reported previously, the mRNA expression was measured by microarray. The probe set intensities were quantified using the GeneChip Operating Software (GCOS) and normalized with GCRMA (GeneChip Robust Multiarray Averaging) using Array Assist Software.

\section{IHC staining and evaluation}

Tissue microarray on glass slides was deparaffinized in xylene, dehydrated in graded ethanol and subjected to antigen retrieval in boiling citrate buffer $(0.01 \mathrm{M}, \mathrm{pH}$ 6.0). Then, the sections were blocked by UltraVision Hydrogen Peroxide Block (Thermo Scientific, CA, USA) and UltraVision Protein Block (Thermo Scientific), followed by primary antibody incubation (1:1000; Abcam, USA). UltraVision Quanto Detection System horseradish peroxidase (HRP) Polymer (Thermo Scientific) and DAB Quanto (Thermo Scientific) were applied staining and hematoxylin was used for counterstaining. We also make an H.E staining of the tissue microarray to discriminate tumor part and other part. The staining in gastric cancer cells, not the stromal part of the cancer tissue, were calculated. The staining intensity was categorized as follows: 0, negative; 1, weak; 2, moderate; and 3, strong. Depending on the staining extent, the area was categorized as follows: $0,<5 \% ; 1,5-25 \% ; 2,26-50 \% ; 3$, $51-75 \%$; and $4,>75 \%$. The staining score was calculated by combining staining intensity and area, yielding a series of results ranging from 0 to 12 according to our previous report [16]. The best cut off point was determined by the ROC analysis.

\section{Statistical analysis}

Analyses were performed with SPSS 19.0 (SPSS Inc., Chicago, IL) and R software version 3.0.2 and the "rms" package ( $\mathrm{R}$ Foundation for Statistical Computing, Vienna, Austria). Chi-square test was used to compare categorical variables. Cox proportional hazards model was used to perform univariate and multivariate analysis. Kaplan-Meier analysis was used to determine the survival and log-rank test was used to compare the patient survival between subgroups. The area under the receiver operating characteristic curves (AUC) at different cut-off values of OS time was calculated to determine the optimal cut-off value of the tetranectin expression 
in tumors. Nomogram was created by $\mathrm{R}$ software using "rms" package. Calibration plots were generated to examine the performance characteristics of the predictive nomogram. The Harrell's concordance index (C-Index) and Akaike information criterion (AIC) were used to measure the prognostic accuracy. All statistical analyses were two-sided and $P<0.05$ was regarded as statistically significant.

\section{Results}

\section{The expression of tetranectin in gastric cancer}

We first screened differentially expressed members of C-type lectin family in the TCGA-STAD dataset. Results demonstrated that among the various members of CLEC family, the mRNA expression of $c l e c 3 b$ in gastric cancer tissues displays the most significant fold-change by comparing with that in normal gastric mucosa (Table 1).Statistical analysis also indicated that the tetranectin expression was remarkably down-regulated in gastric cancer tissues in TCGA $(\mathrm{P}<0.001)$, GSE27342 $(\mathrm{P}<0.001)$ and GSE13911 ( $\mathrm{P}<0.001)$ datasets (Fig. 1a-c).

Table 1. Differentially expressed members of CLEC family in TCGA dataset.

\begin{tabular}{lll}
\hline Gene & Log FC & P-value \\
\hline clec1a & -0.421 & 0.006 \\
clec $2 b$ & -0.603 & 0.000 \\
clec $2 d$ & 0.344 & 0.015 \\
clec $2 l$ & 0.238 & 0.710 \\
clec $3 a$ & -2.617 & 0.000 \\
clec $3 b$ & -3.490 & 0.000 \\
clec4a & 0.482 & 0.027 \\
clec $4 d$ & 0.482 & 0.202 \\
clec4e & 0.584 & 0.083 \\
clec4f & -1.209 & 0.000 \\
clec4g & -2.273 & 0.000 \\
clec5a & 2.345 & 0.000 \\
clec7a & 0.574 & 0.015 \\
clec $9 a$ & -1.324 & 0.000 \\
clec10a & -2.075 & 0.000 \\
clec11a & 0.334 & 0.214 \\
clec12a & 0.477 & 0.113 \\
clec14a & -0.403 & 0.007 \\
clec16a & 0.103 & 0.255 \\
clec17a & -0.118 & 0.902 \\
clec18a & 0.544 & 0.060 \\
clec18b & 0.279 & 0.427 \\
clecl 1 & -0.407 & 0.318 \\
\hline
\end{tabular}

Abbreviations: FC, fold change.

Next we investigated the expression of tetranectin in 328 cases of gastric cancer with tissue microarray. The representative staining of tetranectin in tumor tissues and adjacent normal tissues were shown in Figure 1d. In comparison to the H.E staining, it revealed that the expression of tetranectin was mostly in the cytoplasm of gastric epithelial cells, not the stromal part of the cancer tissue (Figure S1). Statistical analysis confirmed that tetranectin staining score in tumor tissue was significantly lower than that in normal gastric epithelium $(\mathrm{P}<0.001)$ (Fig. 1e). These results suggest that the expression of tetranectin is down-regulated in gastric cancer.

\section{Correlation between tetranectin expression and clinicopathological features in gastric cancer}

According to the results conducted by receiver operating characteristic (ROC) curve analysis, IHC score of 6 was determined as the cut-off to dichotomize the patients into tetranectin low expression group (score, $0-6 ; \mathrm{n}=221$ ) and tetranectin high expression group (score, $7-12 ; \mathrm{n}=107$ ). The correlations between tetranectin expression and clinicopathological features in 328 gastric cancer samples were also explored with the chi-square test (Table 2). Among the variables, higher expression of tetranectin was positively associated with tumor invasion $(\mathrm{P}=0.013)$, lymph node metastasis $(\mathrm{P}=$ $0.005)$, advanced TNM stage $(P=0.003)$ and younger age $(\mathrm{P}=0.024)$. No other clinicopathological variables showed a significant difference between tetranectin high and low expression group. We also assessed the expression of tetranectin among different tissue components in the same patient. It showed an increasing expression of tetranectin in invasive part than the normal and in situ part (Figure S2).

\section{Prognostic value of tetranectin expression in gastric cancer}

We next explored the relationship between tetranectin expression and overall survival (OS) in patients with gastric cancer after gastrectomy using Kaplan-Meier analysis. The results demonstrated that high tetranectin expression was associated with poor OS $(\mathrm{P}<0.001)$ (Fig. 2a). To further evaluate the efficiency of tetranectin expression in stratifying patients with different TNM stages, we divided the patients into early (I-II) and advanced (III- IV) groups. In both TNM I-II and TNM III-IV groups, tetranectin expression showed statistically significant value in predicting the outcomes of gastric cancer patients (Fig. 2b, c). These data suggest that tetranectin expression is correlated with OS for patients with gastric cancer.

Tetranectin expression is identified as an independent prognosticator in patients with gastric cancer

Univariate Cox regression analysis was conducted to identify the prognostic significance of clinicopathological factors for OS. Lauren 
classification $(\mathrm{P}<0.001)$, $\mathrm{T}$ classification $(\mathrm{P}<0.001), \mathrm{N}$ classification $(\mathrm{P}<0.001)$, distant metastasis $(\mathrm{P}=0.004)$, TNM stage $(\mathrm{P}<0.001)$ and tetranectin expression $(\mathrm{P}<$ 0.001 ) were defined as risk factors that may affect the OS of gastric cancer patients (Table 3). After adjustment of covariate factors by using multivariate Cox analysis, we identified Lauren classification $(\mathrm{P}<$ $0.001)$, T classification $(\mathrm{P}=0.006), \mathrm{N}$ classification $(\mathrm{P}=$ $0.001)$, distant metastasis $(\mathrm{P}=0.001)$, and tetranectin expression $(\mathrm{P}<0.001)$ as independent prognostic factors for OS (Table 3).

\section{Combination of tetranectin expression with TNM stage generates a better predictive model for overall survival of gastric cancer patients}

To improve the prognostic accuracy for OS of patients with gastric cancer after gastrectomy, we combined tetranectin expression and TNM staging system together to generate a predictive model. ROC curve analysis showed that the combination of tetranectin and TNM stage revealed better prognostic value (AUC [95\% CI], 0.749 [0.686-0.813]) than tetranectin expression alone (AUC [95\% CI], 0.657 [0.592-0.721]) or TNM stage alone (AUC [95\% CI], 0.672 [0.598-0.747]) with statistical significance (Fig. 3a). In addition, the AIC was 2647.21 when estimated according to TNM stage alone, and it decreased to 2595.652 when estimated in combination with tetranectin expression. The C-index was 0.635 when estimated according to TNM stage alone, and it increased to 0.700 when tetranectin expression was added (Table 4). a

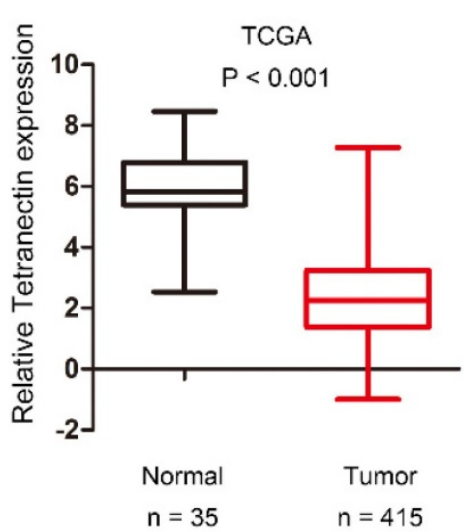

d

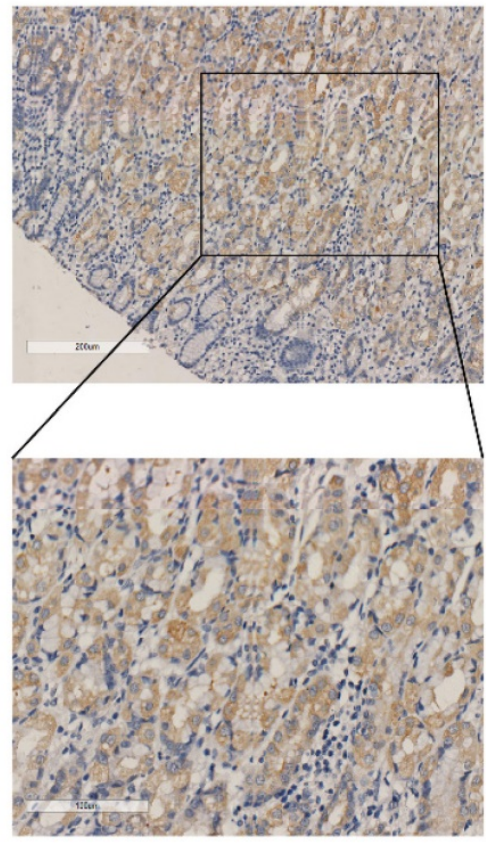

Normal b
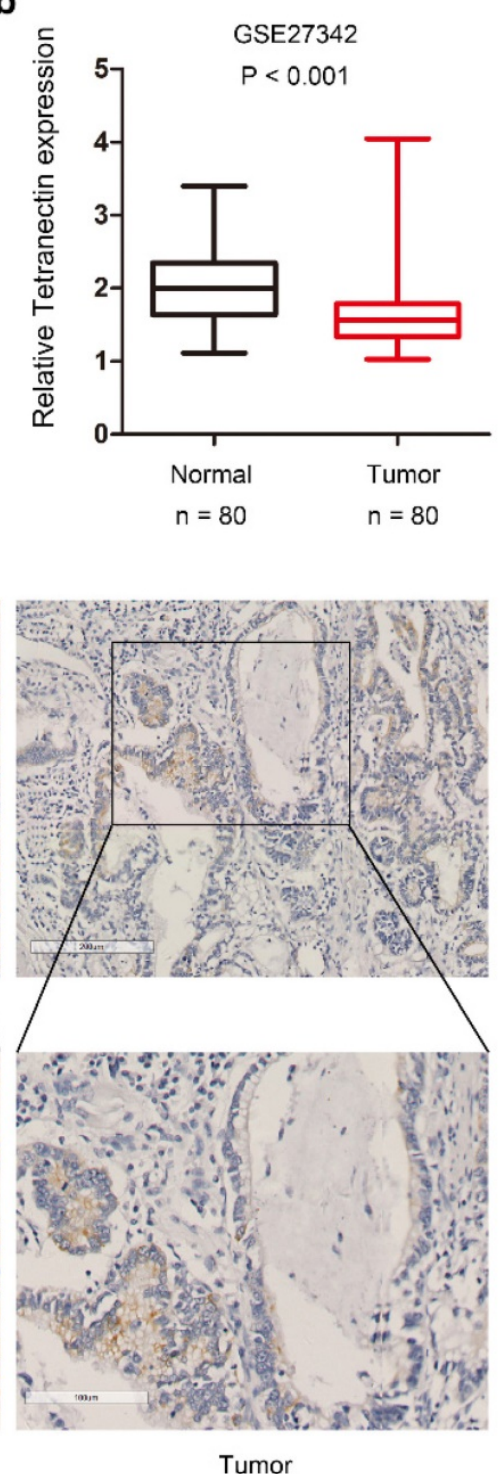

C

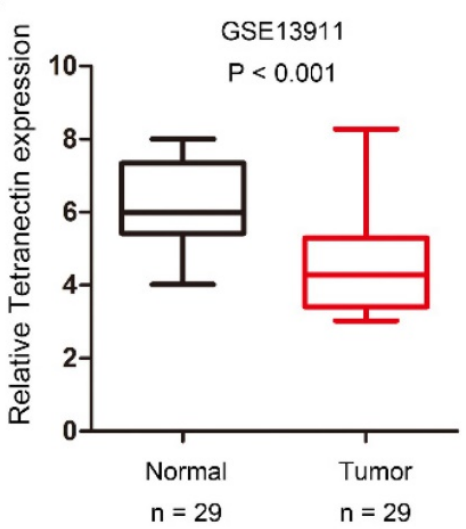

$\mathbf{e}$

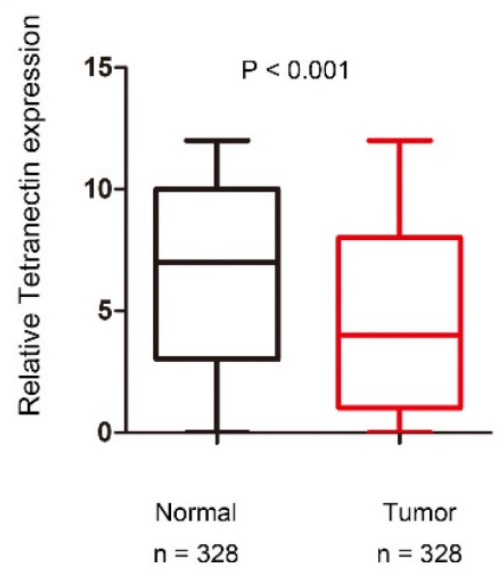

Figure 1. The expression of tetranectin in gastric cancer tissues. Relative expression of tetranectin mRNA in gastric cancer and normal gastric epithelium in TCGA (a), GSE27342 (b), GSE13911 (c). (d) Representative IHC staining images of tetranectin and its regional magnification in gastric cancer tissues and normal tissues. (e) $\mathrm{IHC}$ score of tetranectin expression in gastric cancer tissues and normal tissues. 
We also conducted a predictive nomogram by combing all independent prognostic factors after multivariate Cox regression analysis (Fig. $3 b$ ). In the nomogram, a higher total point predicts a worse prognosis. The total point was calculated by adding the score of Lauren classification, $\mathrm{T}$ stage, $\mathrm{N}$ stage, $\mathrm{M}$ stage and tetranectin expression respectively. The calibration curve for predicted 5-year OS performed well with the ideal mode (Fig. 3c). We next stratified the 328 patients with gastric cancer into 3 groups according to the points calculated by the nomogram: low-risk $(<25$ th percentile), intermediate-risk (25th-75th percentile), and high-risk ( $>$ 75th percentile) groups (Fig. 3d). The results showed that the nomogram could effectively discriminate the risk of OS in patients with gastric cancer.

\section{Discussion}

It is well recognized that gastric cancer is a highly heterogeneous disease with poor clinical outcome. To provide a predictive model for patients with gastric cancer, traditional TNM staging system and the Lauren classification were commonly used in clinical practice $[17,18]$. Nevertheless, these predictive models still have a limit in their ability to discriminate a subset of patients when referring to the molecular heterogeneity of gastric cancer [19]. Classification of gastric carcinomas based on molecular subtypes are supposed to be used in the near future to determine prognosis and to customise treatment [1]. Recently, the Asian Cancer Research Group (ACRG) applied gene expression profiling and defined four distinct gastric cancer molecular subtypes that are associated with distinct genomic alterations, survival outcome and recurrence patterns after surgery [20], which shows prognostic value of gastric cancer by using molecular approaches. Moreover, molecular classification based on human epidermal growth factor receptor 2 (HER2; also known as ERBB2) status has been introduced in gastric cancers because of therapeutic implications [21]. In our study, we found a significant association between high tetranectin expression and poor
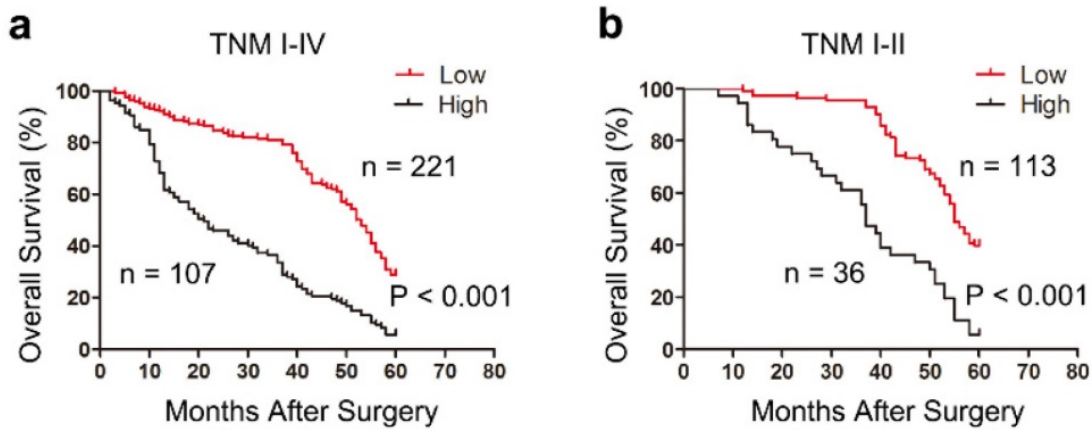

prognosis in gastric cancer patients following surgery, and the molecular mechanism and potential functions of tetranectin in gastric cancer needs further investigation.

Table 2. Correlation between tetranectin expression and clinicopathological characteristics in patients with gastric cancer.

\begin{tabular}{|c|c|c|c|c|c|c|}
\hline $\begin{array}{l}\text { Clinicopathological } \\
\text { Characteristics }\end{array}$ & No. & $\begin{array}{l}\text { Tetranectin } \\
\text { low }\end{array}$ & $\%$ & $\begin{array}{l}\text { Tetranectin } \\
\text { high }\end{array}$ & $\%$ & $P$-value \\
\hline & 328 & & & & & \\
\hline Gender & & & & & & 0.596 \\
\hline Male & 215 & 147 & 68 & 68 & 32 & \\
\hline Female & 113 & 74 & 65 & 39 & 35 & \\
\hline Age & & & & & & 0.024 \\
\hline$<60$ & 158 & 116 & 73 & 42 & 27 & \\
\hline$\geqq 60$ & 170 & 105 & 62 & 65 & 38 & \\
\hline Tumor location & & & & & & 0.739 \\
\hline Cardiac & 40 & 25 & 63 & 15 & 38 & \\
\hline Body & 82 & 57 & 70 & 25 & 30 & \\
\hline Pylorus & 206 & 139 & 67 & 67 & 33 & \\
\hline $\begin{array}{l}\text { Tumor } \\
\text { differentiation }\end{array}$ & & & & & & 0.703 \\
\hline Well & 13 & 10 & 77 & 3 & 23 & \\
\hline Moderate & 51 & 33 & 65 & 18 & 35 & \\
\hline Poor & 264 & 178 & 67 & 86 & 33 & \\
\hline $\begin{array}{l}\text { Lauren } \\
\text { classification }\end{array}$ & & & & & & 0.266 \\
\hline Diffused Type & 137 & 95 & 69 & 42 & 31 & \\
\hline Intestinal Type & 180 & 121 & 67 & 59 & 33 & \\
\hline Mixed Type & 11 & 5 & 45 & 6 & 55 & \\
\hline $\mathrm{T}$ & & & & & & 0.013 \\
\hline $\mathrm{T} 1$ & 71 & 58 & 82 & 13 & 18 & \\
\hline $\mathrm{T} 2$ & 30 & 21 & 70 & 9 & 30 & \\
\hline $\mathrm{T} 3$ & 84 & 57 & 68 & 27 & 32 & \\
\hline $\mathrm{T} 4$ & 143 & 85 & 59 & 58 & 41 & \\
\hline $\mathrm{N}$ & & & & & & 0.005 \\
\hline No & 103 & 77 & 75 & 26 & 25 & \\
\hline N1 & 55 & 41 & 75 & 14 & 25 & \\
\hline N2 & 64 & 44 & 69 & 20 & 31 & \\
\hline N3 & 106 & 59 & 56 & 47 & 44 & \\
\hline M & & & & & & 0.137 \\
\hline M0 & 318 & 217 & 68 & 102 & 32 & \\
\hline M1 & 10 & 4 & 40 & 5 & 50 & \\
\hline TNM stages & & & & & & 0.003 \\
\hline I & 73 & 61 & 84 & 12 & 16 & \\
\hline II & 76 & 52 & 68 & 24 & 32 & \\
\hline III & 170 & 104 & 61 & 66 & 39 & \\
\hline IV & 9 & 4 & 44 & 5 & 56 & \\
\hline
\end{tabular}

Figure 2. Kaplan-Meier survival analysis showing the relationship between tetranectin expression and overall survival in all patients (a), patients at TNM I-II stage (b) and patients at TNM III -IV stage (c). 
a

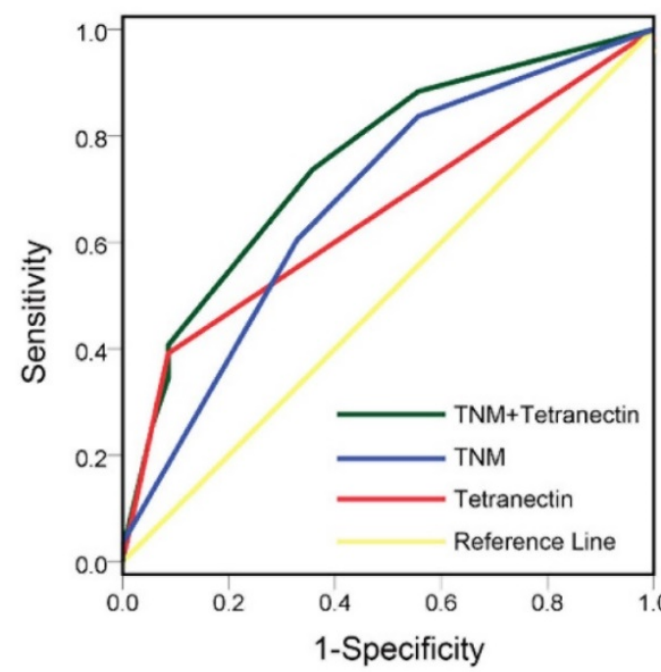

AUC $\quad 95 \% \mathrm{Cl} \quad$ P-value

TNM+Tetranectin $\quad 0.749 \quad 0.686-0.813$

$\begin{array}{llll}\text { TNM } & 0.672 & 0.598-0.747 & 0.003\end{array}$

$\begin{array}{llll}\text { Tetranectin } & 0.657 & 0.592-0.721 & <0.001\end{array}$

b

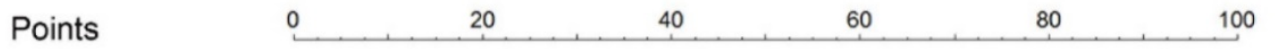

Lauren

classification

Intestinal Mixed Diffused

$\mathrm{T}$ stage

$\mathrm{N}$ stage

M stage

Tetranectin expression
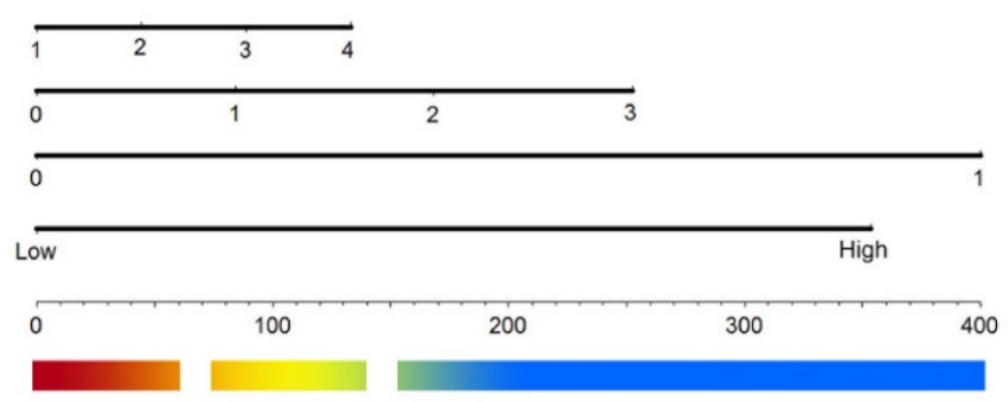

Low risk Medium risk High risk

3-year survival

5-year survival

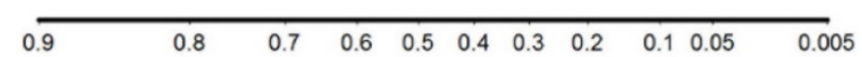

C

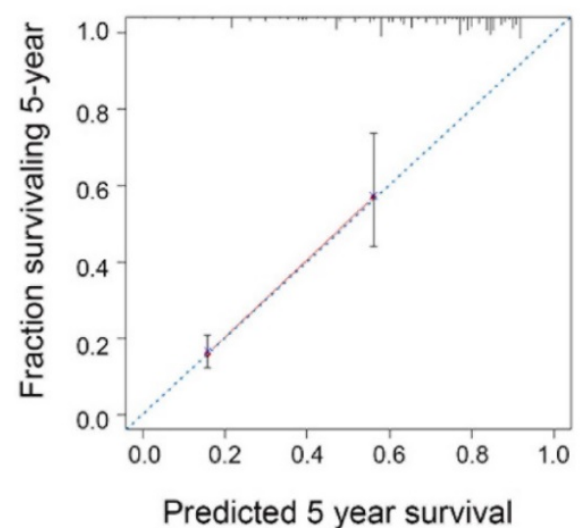

d

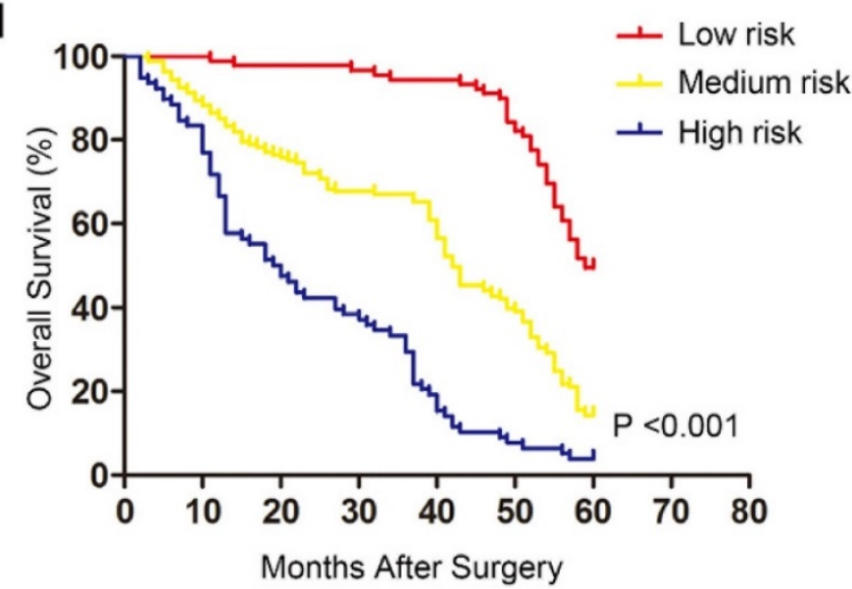

Figure 3. ROC curve and nomogram for predicting prognosis in patients with gastric cancer. (a) ROC curve analysis of the sensitivity and specificity for the predictive value of the TNM model, the tetranectin model, and the combined TNM and tetranectin stratification model. (b) Predictive nomogram for 3-year and 5-year overall survival was generated by combining proven independent prognostic factors including Lauren classification, $\mathrm{T}$ stage, $\mathrm{N}$ stage, $\mathrm{M}$ stage and tetranectin expression. (c) Calibration plot for nomogram predicting observed 5-year survival rate. Gray Line: ideal model; vertical bars, $95 \%$ confident interval. (d) Kaplan-Meier curves of overall survival based on risk score calculated by nomogram. $P$-value was assessed by log-rank test. 
Table 3. Univariate and multivariate Cox regression analysis of clinicopathological characteristics influencing the overall survival of gastric cancer patients.

\begin{tabular}{|c|c|c|c|c|c|c|}
\hline \multirow[t]{2}{*}{ Variables } & \multicolumn{3}{|l|}{ Univariate } & \multicolumn{3}{|l|}{ Multivariate } \\
\hline & Hazard ratio & $(95 \% \mathrm{CI})$ & $P$-value & Hazard ratio & $(95 \% \mathrm{CI})$ & $P$-value \\
\hline $\begin{array}{l}\text { Gender: } \\
\text { male versus female }\end{array}$ & 0.954 & $0.737-1.236$ & 0.723 & & & \\
\hline $\begin{array}{l}\text { Age (Year): } \\
\geq 60 \text { versus }<60\end{array}$ & 1.234 & $0.966-1.576$ & 0.093 & & & \\
\hline Tumor Location: cardia + body versus antrum & 0.856 & $0.666-1.1$ & 0.224 & & & \\
\hline $\begin{array}{l}\text { Differentiation: } \\
\text { poorly +moderately } \\
\text { versus well }\end{array}$ & 1.973 & $0.931-4.182$ & 0.076 & & & \\
\hline Lauren classification: diffused + mixed versus intestinal & 2.007 & $1.564-2.577$ & $<0.001$ & 1.96 & $1.516-2.534$ & $<0.001$ \\
\hline $\begin{array}{l}\text { T classification: } \\
\text { T2-4 versus T1 }\end{array}$ & 2.492 & $1.764-3.521$ & $<0.001$ & 1.754 & $1.173-2.623$ & 0.006 \\
\hline $\begin{array}{l}\mathrm{N} \text { classification: } \\
\mathrm{N} 2+\mathrm{N} 3 \text { versus } \mathrm{N} 0+\mathrm{N} 1\end{array}$ & 2.047 & $1.596-2.624$ & $<0.001$ & 1.779 & $1.286-2.461$ & 0.001 \\
\hline $\begin{array}{l}\text { M classification: } \\
\text { M1 versus M0 }\end{array}$ & 2.708 & $1.385-5.292$ & 0.004 & 3.381 & $1.685-6.783$ & 0.001 \\
\hline $\begin{array}{l}\text { TNM stage: } \\
\text { III+IV versus I+II }\end{array}$ & 2.055 & $1.599-2.641$ & $<0.001$ & 0.952 & $0.665-1.362$ & 0.787 \\
\hline $\begin{array}{l}\text { Tetranectin expression: } \\
\text { high versus low }\end{array}$ & 3.039 & 2.351-3.927 & $<0.001$ & 2.848 & 2.192-3.702 & $<0.001$ \\
\hline
\end{tabular}

Abbreviations: $\mathrm{P}<0.05$ was considered statistically significant.

Table 4. AIC and Harrell's C-index analysis of the comparison of the predictive accuracies of different models.

\begin{tabular}{llll}
\hline Model & AIC & C-index & $95 \%$ CI \\
\hline TNM & 2647.21 & 0.635 & $0.605-0.666$ \\
Tetranectin & 2628.349 & 0.632 & $0.604-0.659$ \\
TNM+ Tetranectin & 2595.652 & 0.700 & $0.671-0.730$ \\
Nomogram & 2559.376 & 0.741 & $0.715-0.767$ \\
\hline
\end{tabular}

Abbreviations: AIC, Akaike information criterion; C-index, Harrell's concordance index; CI, confident interval.

Tetranectin belongs to the family of C-type lectins that bind specifically to kringle four of plasminogen [22]. C-type lectins are a kind of carbohydrate-binding molecules whose functions span many areas of immunity and homeostasis [23]. Recent studies have suggested the potential correlation between C-type lectins and carcinogenesis including gastric cancer [24]. Our recent research also indicated that C-type lectin receptor CLEC-2 suppressed AKT signaling and invasive activities of gastric cancer cells by blocking expression of PI3K subunits [25]. The expression pattern of tetranectin has also been described in several types of human carcinomas. Serum tetranectin have shown great distinguishing ability between autoimmune pancreatitis and pancreatic cancer [26], as well as benign ovarian lesions and malignant ovarian tumors [27]. However, the role of intratumoral tetranectin expression as a tumor prognostic factor remains controversial. High tetranectin expression were found to be associated with poor survival in breast cancer [10] and bladder cancer [11], while positive expression of tetranectin predicted a favorable survival prognosis in women diagnosed with ovarian cancer [13].

The mechanisms of tetranectin in human malignancies are being explored. It has been reported that tetranectin plays an important role in regulating the fibrinolysis and proteolytic procedures by binding to kringle four of circulating plasminogen to enhance activation of plasminogen into plasmin [28]. Although the precise biological function of tetranectin has not yet been clarified, it shows co-localization with plasminogen at the invasion front of melanomas, suggesting a role in cancer invasion and metastases [29]. Increased diffuse cytoplasmic immunoreactivity of urokinase-type plasminogen activator (u-PA) were also found in neoplastic columnar epithelial cells of colon carcinoma while intense immunoreactivity for tetranectin in the stroma surrounding the tumor cells simultaneously [30]. As u-PA is found up-regulated in gastric cancer cells to enhance invasiveness [31, 32], tetranectin were hypothesized to combine with, or get close proximity to u-PA to enhance tumor associated proteolytic activity [30]. Since proteolytic activity is essential for tumor growth, invasion, and metastasis [33], it is in accordance with our observations that high expression of tetranectin was associated with increased invasiveness and metastasis in gastric cancer. These data imply that tetranectin may play an important role in proteolytic activity to enhance invasion and metastasis of gastric cancer, while the exact mechanisms still needs further investigation.

Although the clinical significance of tetranectin in gastric cancer has been presented in our study, some limitations of this study should be acknowledged. First, the number of patients enrolled in this study was relatively small. Second, our data lack the serum levels of tetranectin. Third, single cohort seems to be inadequate to reach greater reliability. Thus, a large, multi-center, prospective 
data is needed to validate these results and more efforts need to be exerted in the future studies.

In summary, our data indicated that high intratumoral expression of tetranectin correlated with tumor invasion and metastasis of gastric cancer, and was identified as an independent inferior prognostic factor of OS for patients after gastrectomy. Incorporation of tetranectin expression with TNM staging system or other predictive models might add some prognostic information for patients' survival.

\section{Supplementary Material}

Supplementary figures.

http://www.jcancer.org/v08p3623s1.pdf

\section{Acknowledgements}

This study was funded by grants from National Natural Science Foundation of China (81672925, $31670806,81572317,31370808,81302259,31500645$, 31630088). All these study sponsors have no roles in the study design, in the collection, analysis, and interpretation of data.

\section{Competing Interests}

The authors have declared that no competing interest exists.

\section{References}

1. Van Cutsem E, Sagaert X, Topal B, Haustermans K, Prenen H. Gastric cancer. The Lancet. 2016.

2. Ferlay J, Steliarovafoucher E, Lortettieulent J, Rosso S, Coebergh JW, Comber $\mathrm{H}$, et al. Cancer incidence and mortality patterns in Europe: Estimates for 40 countries in 2012. European Journal of Cancer. 2015; 51: 1201-2.

3. Chen W, Zheng R, Baade PD, Zhang S, Zeng H, Bray F, et al. Cancer statistics in China, 2015. Ca A Cancer Journal for Clinicians. 2016; 66: 115-32.

4. Duraes C, Almeida GM, Seruca R, Oliveira C, Carneiro F. Biomarkers for gastric cancer: prognostic, predictive or targets of therapy? Virchows Arch. 2014; 464: 367-78.

5. Clemmensen I, Petersen LC, Kluft C. Purification and characterization of a novel, oligomeric, plasminogen kringle 4 binding protein from human plasma: tetranectin. European Journal of Biochemistry. 1986; 156: 327-33.

6. Iba K, Abe Y, Chikenji T. Delayed fracture healing in tetranectin-deficient mice. Journal of Bone and Mineral Metabolism. 2013; 31: 399-408.

7. Iba K, Hatakeyama N, Kojima T, Murata M, Matsumura T, Wewer UM, et al. Impaired cutaneous wound healing in mice lacking tetranectin. Wound Repair \& Regeneration. 2009; 17: 108-12.

8. Wang ES, Zhang XP, Yao HB, Wang G, Chen SW, Gao WW, et al. Tetranectin knockout mice develop features of Parkinson disease. Cellular Physiology \& Biochemistry International Journal of Experimental Cellular Physiology Biochemistry \& Pharmacology. 2014; 34: 277-87.

9. Begum FD, Høgdall E, Christensen IJ, Kjaer SK, Blaakaer J, Christensen L, et al. Serum tetranectin is a significant prognostic marker in ovarian cancer patients. Acta Obstetricia Et Gynecologica Scandinavica. 2010; 89: 190-8.

10. Obrist P, Spizzo G. Aberrant tetranectin expression in human breast carcinomas as a predictor of survival. Journal of Clinical Pathology. 2004; 57: 417-21.

11. Brunner A, Ensinger C, Christiansen M, Heiss S, Verdorfer I, Mikuz G, et al. Expression and prognostic significance of Tetranectin in invasive and non-invasive bladder cancer. Virchows Arch. 2007; 450: 659-64.

12. Arellano-Garcia ME, Li R, Liu X, Xie Y, Yan X, Loo JA, et al. Identification of tetranectin as a potential biomarker for metastatic oral cancer. Int J Mol Sci. 2010; 11: 3106-21.

13. Heeran MC, Rask L, Hogdall CK, Kjaer SK, Christensen L, Jensen A, et al. Tetranectin positive expression in tumour tissue leads to longer survival in Danish women with ovarian cancer. Results from the 'Malova' ovarian cancer study. APMIS. 2015; 123: 401-9.

14. Zhu Y, Qiu P, Ji Y. TCGA-Assembler: open-source software for retrieving and processing TCGA data. Nature Methods. 2014; 11: 599-600.
15. Robinson MD, Oshlack A. A scaling normalization method for differential expression analysis of RNA-seq data. Genome Biology. 2010; 11: 1-9.

16. Chen L, Min L, Wang X, Zhao J, Chen H, Qin J, et al. Loss of RACK1 Promotes Metastasis of Gastric Cancer by Inducing a miR-302c/IL8 Signaling Loop. Cancer Res. 2015; 75: 3832-41.

17. Edge SB, Compton CC. The American Joint Committee on Cancer: the 7th Edition of the AJCC Cancer Staging Manual and the Future of TNM. Annals of Surgical Oncology. 2010; 17: 1471.

18. Turner ES, Turner JR. Expanding the Lauren classification: a new gastric cancer subtype? Gastroenterology. 2013; 145: 505-8.

19. Stock M, Otto F. Gene deregulation in gastric cancer. Gene. 2005; 360: 1-19.

20. Cristescu R, Lee J, Nebozhyn M, Kim KM, Ting JC, Wong SS, et al. Molecular analysis of gastric cancer identifies subtypes associated with distinct clinical outcomes. Nature Medicine. 2015; 21: 449.

21. Ajani JA, D'Amico TA, Almhanna K, Bentrem DJ, Chao J, Das P, et al. Gastric Cancer, Version 3.2016; Clinical Practice Guidelines in Oncology. 2016.

22. Holtet TL, Graversen JH, Thøgersen HC, Etzerodt M, Clemmensen I. Tetranectin, a trimeric plasminogen-binding C-type lectin. Protein Science A Publication of the Protein Society. 1997; 6: 1511.

23. Kutikhin AG, Yuzhalin AE. C-type lectin receptors and RIG-I-like receptors: new points on the oncogenomics map. Cancer Management \& Research. 2012; 4: 39 .

24. Castaño-Rodríguez N, Kaakoush NO, Mitchell HM. Pattern-recognition receptors and gastric cancer. Frontiers in Immunology. 2014; 5: 336.

25. Wang L, Yin J, Wang X, Shao M, Duan F, Wu W, et al. C-type Lectin-like Receptor 2 Suppresses AKT Signaling and Invasive Activities of Gastric Cancer Cells by Blocking Expression of PI3K Subunits. Gastroenterology. 2016; 150: 1183-95.e16.

26. Felix K, Hauck O, Fritz S, Hinz U, Schnolzer M, Kempf T, et al. Serum protein signatures differentiating autoimmune pancreatitis versus pancreatic cancer. PLoS One. 2013; 8: e82755.

27. Begum FD, Hogdall E, Kjaer SK, Blaakaer J, Christensen IJ, Christensen L, et al. Preoperative serum tetranectin, CA125 and menopausal status used as single markers in screening and in a risk assessment index (RAI) in discriminating between benign and malignant ovarian tumors. Gynecol Oncol. 2009; 113: 221-7.

28. Iba K, Hatakeyama N, Kojima T, Murata M, Matsumura T, Wewer UM, et al. Impaired cutaneous wound healing in mice lacking tetranectin. Wound Repair Regen. 2009; 17: 108-12

29. Wang ES, Sun Y, Guo JG, Gao X, Hu JW, Zhou L, et al. Tetranectin and apolipoprotein A-I in cerebrospinal fluid as potential biomarkers for Parkinson's disease. Acta Neurol Scand. 2010; 122: 350-9.

30. Verspaget HW, Clemmensen I, Ganesh S, Christensen L, Sier CF, Griffioen G, et al. Tetranectin expression in human colonic neoplasia. Histopathology. 1994; 25: 463-7.

31. Lee DH, Yang Y, Lee SJ, Kim KY, Koo TH, Shin SM, et al. Macrophage inhibitory cytokine-1 induces the invasiveness of gastric cancer cells by up-regulating the urokinase-type plasminogen activator system. Cancer Research. 2003; 63: 4648-55.

32. Khoi PN, Xia Y, Lian S, Kim HD, Kim dH, Joo YE, et al. Cadmium induces urokinase-type plasminogen activator receptor expression and the cell invasiveness of human gastric cancer cells via the ERK-1/2, NF- $\mathrm{kB}$, and AP-1 signaling pathways. International Journal of Oncology. 2014; 45: 1760-8.

33. Markus G. The Role of Hemostasis and Fibrinolysis in the Metastatic Spread of Cancer. Seminars in Thrombosis and Hemostasis; 1984. 